\title{
EFFECT OF FREE RADICAL/ANTIOXIDANTS IN HUMAN BODY
}

\author{
IRIN. D. PRAVEEN
}

Vice Principal, Venkateswara Nursing College, Chennai, Tamil Nadu, India

\begin{abstract}
The presence of one unpaired electron in a molecule is called free radical. When oxygen interacts with certain molecules in chemical reaction it produces atoms or group of atoms with unpaired electrons called free radicals. These are highly reactive in nature, they start a chain reaction which adversely alters the lipids, proteins, and DNA structure of a cell which triggers a number of human diseases. Cells may function poorly or die if this occurs.

The body has natural defense system of antioxidants which can safely interact with free radicals and terminate the chain reaction before vital molecules are damaged thereby preventing the effect of free radical. Although there are several enzyme systems within the body that scavenge free radicals, the principle micronutrient (vitamin) antioxidants are vitamin $E$, beta-carotene, and vitamin $C$. The body cannot produce these micronutrients on its own so they must be supplemented through diet. The present review provides an overview of oxidative stress-mediated cellular damages and role of dietary antioxidants to counter its effect.

KEYWORDS: Ageing, Antioxidant, Free Radicals\& Oxidative Stress
\end{abstract}

Received: Feb 07, 2018; Accepted: Feb 28, 2018; Published: Apr 07, 2018; Paper Id.: IJMPSAPR201810

\section{INTRODUCTION}

The unpaired electron of free radical combines with other electrons to form a pair and becomes highly reactive, this process is called oxidation, they steal electrons from the combining molecules and alters the fats, proteins, cell membranes, DNA molecule of the combining cell altering the basic structure of the affected molecule. Antioxidant the alternative inhibitor is comprised of atoms with excess electrons that it can "donate" to stabilize the free radical and stop the effect fashioned throughout its chain reaction. Thereby it helps to slow down aging or stop the aging process because they prevent cell injury, which occurs with aging. Certain vitamins contain "antioxidant especially vitamin A, C, E and B. They are not produced naturally in the body but must be consumed through diet or supplementation.

\section{FORMATION OF FREE RADICALS}

Cell metabolism in human life needs an essential and vital component called oxygen. Free radicals are a natural byproduct of cellular metabolism. ${ }^{[2]}$ Apart from cellular metabolism the other ways in which the body produces are inflammation, stress, illness, and aging. Environmental factors such as toxic metals, air, and water pollution, consuming alcohol, cigarette smoking, exposure to radiation and industrial chemicals, medications daily stress - emotional and physical, ozone depletion, UV rays from the sun, industrial chemicals, processed foods, etc are other sources of free radical.[3] Free radicals can also be formed in non-enzymatic reactions of oxygen with organic compounds as well as those initiated by ionizing reactions 
Avoiding or preventing exposure to free radicals is not attainable. Moreover, it is not harmful always as it is helpful to the human body at minimum exposure, the body, uses them in defend itself against pathogens. ${ }^{[4]}$

Excess free radicals within the body, results to aerophilous stress. The body is beneath constant attack of aerophilous stress during which the atom of the chemical element present in the body splits into single atoms with unpaired electrons referred to as free radicals which seek out other alternative electrons from neighboring cells to combine with it and become a pair. This causes injury to the opposite cell, proteins, and DNA molecules. The exposure of free radical causes certain illness like Alzheimer's brain disease, cancer, and atherosclerosis. They also conjointly cause aging, due to the gradual accumulation of free-radical damage.

Free radicals are the natural byproducts obtained from chemical changes resulting from metabolism. According to Lauri Wright, free radicals are wasted products from various chemical reactions within the cell and once it gets accumulated in the body harms the cells of the body. Although they are harmful they are essential to life, as it enables the body's ability to turn air and food into chemical energy and also play a crucial part in supporting the immune system, by attacking foreign invaders.

\section{THE DANGER OF FREE RADICALS}

Free radicals initiate series of the chain reaction. The free radical pull an electron from a molecule, which damages it and turns it into a free radical. That molecule, in turn takes, an electron from another molecule, destabilizing it and turning it into a free radical. This continuous damaging effect can eventually disrupt and damage the whole cell. The chain reaction of free radicals may lead to broken cell membranes, which dysfunctions the protective nature of the cell membrane.

Free radicals exist only for a few seconds, but during their fleeting existence, they cause some serious structural damage to the cell. They often leave a huge path of destruction in their wake. Once a molecule is oxidized by the free radical, it often starts a chain reaction of oxidation until an antioxidant stops its ability. That's why free radicals and antioxidants must go hand in hand.

\section{OXIDATIVE STRESS}

The imbalance between free radicals and body's store of antioxidants results in the oxidative damage which is called oxidative stress. According to the free radical theory of aging, the accumulated free radical causes damage to cells and DNA

Leading to aging by accumulative damage to cell structures and connective tissue resulting in the formation of wrinkles, diminished physical capability, poor immune system, susceptibility to disease, and death. ${ }^{[5]}$ Although the radical theory of aging remains arguable, oxidative stress contributes to degenerative conditions such as arthritis, inflammatory disease, cardiovascular disease, Alzheimer's disease, Parkinson's disease, chronic conditions of skin, emphysema, dementia, ulcers, arthritis and more. ${ }^{[1]}$

\section{IMPACT OF EXERCISE ON FREE RADICALS}

Intense aerobic exercise can induce oxidative stress. High-intensity cardio exercise causes chemical reactions by burning fat that produces free radicals which are formed at a faster rate. The concern that intense exercise could cause oxidative stress initiated several studies to assess the effects of antioxidant supplementation for athletes. 
According to American Journal of Clinical Nutrition regular exercise alone is enough to build up antioxidant defenses against the initial exercise-induced oxidative stress. However, supplementing high-intensity exercise with antioxidant supplements produced no beneficial effects. Therefore irregular exercisers and individuals who are excess in weight but who do only a few about of intense physical activity spontaneously may invoke oxidative stress, while those who are consistently active need not worry.

\section{ANTIOXIDANTS AGAINST FREE RADICALS}

The term antioxidant originally was used to refer specifically to a chemical that prevented the consumption of oxygen. These have the capacity to protect the cells against the harmful effects of free radicals as they are a causative factor that influence in bringing about pathological changes and thus producing heart disease, cancer (atherosclerosis) and other diseases. Antioxidants defend the body from injury caused by harmful molecules by binding with free radicals and stop the chain reaction before vital molecules are injured. These antioxidants delay or inhibit cellular damage mainly through their radical scavenging property.

They act as radical scavenger, by donating electron such as hydrogen, electron donor, decomposes peroxide, singlet oxygen quencher, an inhibitor of the selected enzyme reaction, synergist, and metal-chelating agents. They delay or inhibit cellular damage mainly through their free radical scavenging property, terminates the chain reaction thus preventing harmful changes within the molecules. Antioxidants exist in both intracellular and extracellular environment which helps to detoxify the consequences of free radicals.

Some of the antioxidants, including glutathione, ubiquinol, and uric acid, are produced during normal metabolism in the body whereas few lighter antioxidants are found in the diet only. Though several enzymes reactions within the body help to scavenge free radicals, the principle micronutrient vitamins such as vitamin $\mathrm{E}$ ( $\alpha$-tocopherol), vitamin $\mathrm{C}$ (ascorbic acid), and B-carotene contains antioxidants which play a crucial role in dysfunctioning the ability of free radicals. The body cannot manufacture these micronutrients on their own. They must be supplied to the body through food sources.

\section{CONCLUSIONS}

The impact of free radical in the human body brings out adverse biochemical changes leading to dysfunction of structures resulting in disease. But the intake of antioxidants has a counter effect on the radical, by breaking the chain reaction and canceling its harmful effect on the body.

\section{REFERENCES}

1. Aruoma OI. Methodological consideration for characterization for potential antioxidant actions of bioactive components in plants foods. Mutat Res. 2003; 532:9-20. [PubMed]

2. Mohammed AA, Ibrahim AA. Pathological roles of reactive oxygen species and their defence mechanism. Saudi Pharm J. 2004; 12:1-18.

3. Swaroopa. G \& Srinath. D, Antioxidants and Their Roll in Human Health: A Review, International Journal of Agricultural Science and Research (IJASR), Volume 7, Issue 5, September - October 2017, pp. 9-18

4. Bagchi K, Puri S. Free radicals and antioxidants in health and disease. East Mediterranean Health Jr.1998; 4:350-60.

5. Gaziano JM, Glynn RJ, Christen WG, et al. Vitamins $E$ and $C$ in the prevention of prostate and total cancer in men: the Physicians' Health Study II randomized controlled trial.JAMA. 2009; 301(1):52-62. 
6. Goodman M, Bostick RM, Kucuk O, et al. Clinical trials of antioxidants as cancer prevention agents: past, present, and future. Free Radical Biology \& Medicine. 2011; 51(5):1068-1084.

7. Jerome-Morais A, Diamond AM, Wright ME. Dietary supplements and human health: for better or for worse? Molecular Nutrition \&Food Research. 2011; 55(1):122-135.

8. Klein EA, Thompson IM Jr, Tangen CM, et al. Vitamin E and the risk of prostate cancer: the Selenium and Vitamin E Cancer Prevention Trial (SELECT). JAMA. 2011; 306(14):1549-1556. 